\title{
Laurence Wuidar
}

\section{ICONOLOGIE DE LA VOIX: DU SERMON \\ DE VOCE ET VERBO AU TRAITEZ DE LA VOIX ET DES CHANTS}

Parmi les arts, seules la musique et la poésie voient la matière originairement unie à la forme artistique. Le peintre a besoin des couleurs, le sculpteur du marbre ou du bois: les arts visuels opèrent à partir d'une matière préexistante et confèrent une belle forme à cette dernière. Le musicien, à la différence du peintre ou du sculpteur, crée son œuvre dans une coexistence de la matière et de la forme : la matière musicale - le son - est immédiatement forme. Autrement dit, il n'y a pas de division temporelle entre une matière musicale brute et sa mise en belle forme : le son musical revêt immédiatement la beauté de sa forme. C'est ce qu'explique Augustin au livre XII des Confessions et, sur ces bases, I'évêque d'Hippone développe I'analogie bien connue entre création musicale et création divine. De I'Antiquité tardive à la Renaissance, la littérature philosophico-théologique est une source majeure pour I'histoire de I'art. Les Pères de I'Église grecs et latins et à leur suite les théologiens et les philosophes médiévaux débattent du pouvoir des images et de la musique, définissent l'image matérielle et l'image intérieure, pontifient sur les vertus de la musique sacrée et sur les vices de la musique profane, pénètrent dans la psyché humaine pour chercher à y voir ce qui se passe lors de l'écoute musicale, traitent du plaisir sensible, voire sensuel, que provoque la musique, s'intéressent à la voix comme miroir de l'âme ou déchiffrent les significations du silence. Autant de thèmes d'esthétique musicale ante litteram et de philosophie des arts - part intégrante de I'histoire de I'art - dans ses implications éthiques et psychologiques. Il s'agit d'analyser un ensemble de textes qui ne font pas partie de la tradition ni de musicologie ni d'esthétique philosophique (ni classique ni contemporaine) et d'en dégager la spécificité et la pertinence des questions sonores. ${ }^{1}$ Avant I'époque moderne, la voix - ses couleurs, ses altérations, ses significations, ses rapports au son et à la parole, à l'intériorité et à l'extériorité, à l'identité et à l'altérité, au
Matèria, núm. 9, 2015, ISSN 1579-2641, p. 15-38

Recepció: 2-6-2015

Acceptació: 13-7-2015

${ }^{1}$ Il ne s'agit pas ici de se demander comment a été représentée la voix dans les arts visuels - cela a déjà été fait. «Comment représenter le sonore par un médium [la peinture] qui n'affecte que la vue?» s'interroge par exemple Herman Parret dans son essai pour une esthétique de la voix. Herman PARRET, La voix et son temps, Bruxelles, De Boeck \& Larcier, 2002, p. 105. 
2 Dans un récent ouvrage en marge des études musicologiques traditionnelles, Paolo Gozza propose une histoire des rapports entre voix et image. Au départ d'une lecture $d^{\prime}$ Écho et Narcisse, c'est une constellation de sources modernes, de la Phonurgia d'Athanasius Kircher ou la Mechanica hydraulico-pneumatica de Kaspar Schott au xvirème siècle aux traités sur l'imitation dans les arts d'Adam Schmidt à la fin du XviIIème siècle. Paolo GozzA, Imago vocis. Storia di Eco, Milano, Mimesis, 2010.

3 Depuis I'ouvrage de Franco Amerio, Il De musica di sant' Agostino, Torino, Società Editrice Internazionale, 1929, jusqu'à celui de Guillermo PABON, Numerus-proportio en el De Musica de San Agustín: La tradición pitagórico-platónica, Editorial Académica Española, 2011. Dans le contexte présent voir Maria BetTetini: "Ai limiti della materia, tra neoplatonismo e cristianesimo. Per una lettura del 'De musica' di Agostino d'Ippona», Therese FUHRER, Michael ERLER (ed.): Zur Rezeption der hellenistischen Philosophie in der Spätantike, Stuttgart, Franz Steiner Verlag, 1999, p. 123-138; Laura Folli: "Canticum cordis. La musica e l'interiorità nelle Enarrationes in psalmos di Agostino ", Mauro LetTerio (ed.): La musica nel pensiero medievale, Ravenna, Longo Editore, p. 177-183. Pour une bibliographie musicale augustinienne plus complète, voir chant et au silence - , est intimement liée à ce type de sources, et la littérature théologique forge l'iconologie en ce qu'elle représente un des contenants des questions culturelles dont I'importance ne disparaîtra qu'avec les révolutions scientifiques et la naissance de l'esthétique. ${ }^{2}$

Le but de cette contribution est de présenter une série d'étapes pour la construction d'une iconologie de la voix avant l'époque moderne. Ces étapes correspondent à autant de déclinaisons (pistes de recherche) de la voix - dans son rapport à la musique, à l'image et au verbe. Les sources proposées forment un tableau de I'univers sonore et de I'utilisation de I'image musicale chez trois auteurs clés: Augustin (354-430), Grégoire de Nysse (c. 335-394) et Jean Scot Eurigène (c. 810-877). Augustin, dont les Confessions sont autant étudiées que son De musica par les musicologues, ${ }^{3}$ donne dans le De Trinitate une définition de I'image musicale intérieure qui résume ses théories de la connaissance et le rôle de l'image dans ces dernières. Grégoire de Nysse offre une palette du cri au silence dans laquelle la musique occupe une place particulière en ce qu'elle est à l'origine (In inscriptiones Psalmorum) et au terme (Macrine) du désir eschatologique. Jean Scot Eurigène, traducteur de Grégoire et principal philosophe de l'époque carolingienne, pénètre à travers ses spéculations sur la voix dans les méandres de l'identité et de l'altérité alors qu'il traduit la déification de l'être humain, union ultime avec I'Un et conjonction avec I'Altérité, en termes musicaux. Un quatrième auteur, Marin Mersenne (1588-1648), fait faire au lecteur un saut dans le temps, et pourtant le Traitez de la voix et des chants (Paris, 1636) du père de I'Ordre des Minimes est riche d'échos augustiniens et continue à interroger les altérations de la voix - transformée par les passions, miroir identitaire de l'âme et pont vers l'altérité. Entre Grégoire ou Jean Scot et Marin Mersenne, entre le sermon De voce et verbo d'Augustin et le Traitez de la voix et des chants du père minime, la forme du discours change : le contenant des questions sur la voix, sur le son, sur la forme musicale, sur I'harmonie n'est plus le discours de théologie morale ou pastorale, de théologie rationnelle ou mystique, de cosmologie ou d'anthropologie philosophique.

La voix est icône en ce qu'elle est le réceptacle du Verbe et dessine dans la matière sonore les contours identitaires de I'individu. Elle est également iconique en ce qu'elle manifeste l'intériorité (les pensées) et donne les couleurs au chant dont elle est I'origine. Les textes de Grégoire et Jean Scot, Augustin et Mersenne nous plongent dans une analyse philosophique de la voix: son rapport à la matière et à l'esprit, l'interface qu'elle constitue entre l'intériorité et l'extériorité et son rapport à l'altérité - I'ensemble culminant dans ses enjeux musicaux. Cette contribution présente ainsi un ensemble de sources en marge de la culture contemporaine de I'historien 
d'art et du musicologue et qui pourtant l'informe sur son objet d'étude image, musique. La marge délimite et forme I'encadrement du sujet. Et pourtant, la marge n'est pas le texte et n'a donc aucune visée normative.

\section{L'image musicale intérieure chez Augustin}

La dissociation de la vue et de l'ouïe, de l'image et du son, du visage et de la voix est l'inéluctable séparation de Narcisse et d'Écho. Il ne peut que voir, elle ne peut que répéter; il ne peut rien voir d'autre que lui même, elle ne peut rien dire d'autre que ce qu'elle a entendu. Narcisse se perd dans la vision de lui-même, Écho dans la tautologie des paroles qui ne sont ni d'elle ni ne sont entendues (cf. Ovide, Métamorphoses, III, 339-510). La culture chrétienne maintient cette division: il faut croire la Parole (fede ex auditu) sans pouvoir voir ce en quoi I'on croit pour que se réalise la vision future et béate de I'Invisible. La vue est ainsi la perfection de I'ouïe qui n'est que préparation. L'ouie, la parole, le son, la musique sont de simples média voués à disparaître lorsque prendra place la vision de I'Invisible. La perfection appartient à la vision supraterrestre alors que sur terre I'œil ne voit que des reflets de I'Invisible au même titre que I'ouïe qui perçoit des traces de l'Unique dans les multiples harmonies. À chaque sens corporel son objet qui, de par son ordre et sa mesure, est reflet et écho de I'inaccessible divinité. Si le corps divise les traces de I'Unique, l'âme - raison, mémoire, volonté, miroir de la Trinité - les rassemble, tout comme en elle ne sont qu'un la vue et l'ouïe. Les sens intérieurs ne connaissent pas la division, qui est le propre du royaume du corps. La parole et les images ne sont qu'un dans l'âme, où entendre et voir ne se distinguent pas (cf. De Trinitate, XV, 10). Et c'est justement sur ce qui se passe dans I'âme après avoir reçu les sensations, notamment musicales, que s'interroge Augustin dans le De Trinitate.

Le De Trinitate présente d'abord la théorie augustinienne du rapport entre les mots et les choses - entre les paroles évocatrices d'une réalité matérielle et cette réalité perçue par la vue - reprise par de nombreux philosophes médiévaux et renaissants. Dans le livre VIII, Augustin rappelle la nécessité de la médiation analogique comme seul moyen de connaissance des réalités invisibles. Il parle alors de la connaissance que l'être humain a de l'âme de l'autre: quand se meut le corps de l'autre, aucune brèche ne permet d'en voir I'âme (cf. De Trinitate, VIII, 6). Et pourtant, indépendamment de la vue matérielle, l'être perçoit qu'il y a dans cette masse corporelle un principe analogue à celui qui anime la masse de son propre corps. Par analogie, il connaît l'invisible - l'âme motrice du corps
Laurence WUIDAR, La simbologia musicale nei Commenti ai salmi di Agostino, Milano, Mimesis, 2014, p. 17-24. 
4 Traduction française de Raulx De la trinité, Bar-le-Duc, L. Guérin \& Cie, 1868. de I'autre. De là, la division augustinienne bien connue entre phantasia et phantasma: I'image d'une réalité connue directement par la médiation des sens corporels (phantasia) se distingue de la représentation imaginaire d'une réalité connue seulement par la médiation du récit d'un autre (phantasma).

L'image reçue par les sens, image visuelle d'une ville où l'on s'est promené, image sonore d'un morceau musical qu'on a chanté ou écouté, est ensuite conservée dans la mémoire. La représentation imaginaire elle, est une image un peu floue, une image approximative qui s'est formée à l'écoute du récit. S'il était possible de faire sortir la représentation imaginaire de I'âme, l'image ne serait pas fidèle à la réalité. Et si, d'aventure, elle l'était, la représentation imaginaire, la peinture intérieure faite au départ du récit ne pourrait néanmoins pas faire connaître de manière directe la réalité, et l'individu ne pourrait que croire au témoignage des tiers qui lui affirment que sa représentation mentale correspond effectivement à une réalité dont les tiers ont eu une perception et donc une connaissance immédiate - libre de la médiation du récit. La description d'une ville, d'un tableau ou d'une pièce musicale ne peut générer dans I'âme qu'une vision intérieure déformée par manque d'information directe. Au contraire la perception sensible $d^{\prime} u n e$ ville, $d^{\prime} u n$ tableau, $d^{\prime} u n e$ pièce musicale dépose dans la mémoire une image immobile des réalités corporelles perçues par les organes des sens. C'est ce processus d'intériorisation des perceptions (artistiques) que décrit le passage du De Trinitate au départ d'un exemple musical :

Si le rythme d'un son produit par l'art de la musique, en passant à travers le temps, peut être saisi, on peut aussi, en dehors du temps, dans un intime et profond silence, y penser au moins tant qu'on peut I'entendre chanter; cependant ce que le regard de l'âme en aura pris en passant et comme au vol, qu'elle aura ensuite savouré, digéré et mis en réserve dans sa mémoire, elle pourra le ruminer, pour ainsi dire en souvenir et faire passer dans ses connaissances acquises ce qu'elle aura ainsi appris (De Trinitate, XII, 14, 23). ${ }^{4}$

Les images sonores que véhicule la musique se transforment en images immobiles, et la musique acquiert les propriétés des arts visuels: la fluidité musicale cesse son déroulement dans le temps et se fige dans la mémoire. Cette dernière devient la toile où se dessinent les images intérieures reçues par les sens extérieurs et où se fixe l'image musicale. C'est alors I'œil de I'esprit et non I'oreille du corps qui la perçoit. La contemplation de ce tableau intérieur permet à l'être humain d'avoir une meilleure connaissance des messages perçus par les sens. Dans l'intériorité, l'image 
musicale évolue sans nécessité d'espace et de temps. Là, elle perdure audelà de l'espace-temps, d'où la nécessité que ce soit une bonne image. C'est ce que souligne Jean Chrysostome (c. 347-407) un des plus fervents gardiens de la morale notamment musicale des premiers siècles chrétiens. S'il fait du combat à la musique profane - maîtresse de toutes les luxures son cheval de bataille, c'est aux images qu'il s'intéresse dans les homélies sur David et Saül. Dans le De Davide et Saul, Chrysostome note en effet que les images extérieures intériorisées apparaissent jusque dans les rêves. D'où l'importance de montrer aux fidèles de «bonnes images ». Non seulement car la volupté et donc la moralité sont en jeu, mais parce que l'image intérieure a une valeur plus grande que l'image extérieure. En effet, l'individu porte I'image intérieure en lui et, telle une amulette au cou de la mémoire, elle ne le quitte jamais et assure un remède spirituel contre les assauts extérieurs - images, sons, paroles. L'image a alors le même but que la musique, et la théorie de I'image du De Davide et Saul trouve son complément musical dans le commentaire au psaume 4l. Le chant des psaumes est remède contre les tentations, les maux de l'esprit et les invitations à la luxure, le panégyrique sur la musique sacrée - identique à celui de Basile et Ambroise de Milan ${ }^{5}$ - fait de cette dernière le bouclier du fidèle. Protection musicale que reprendront encore les mystiques du XIvème siècle: Richard Rolle (ca. 1300-1349), protégé par une mélodie intérieure qui ne le quitte plus, sera élevé au chœur des anges (cf. Melos amoris, XXX, 101, 7-11).6 Sa cuirasse musicale est aussi sa caution pour I'éternité (cf. Ibid., XXIV, 71, 15-18): prémuni contre toute tentation, muni de son seul chant intérieur (il refuse de chanter les psaumes car la musique sensible le distrait de la musique intérieure et céleste qu'il chante et entend simultanément, cf. Ibid., XLVII, 148, 11-15 et 30-31), le mystique rejoint le chœur angélique dans un raptus musical. Ce raptus musical est aussi un topos des récits chrétiens du trépas.

\section{Mort: cri, murmure, silence chez Grégoire de Nysse}

La musique tient un rôle particulier dans les écrits de Grégoire de Nysse. Indépendamment des nombreuses métaphores musicales qu'il développe sur le plan cosmologique et anthropologique (qui constituent un champ d'étude à part entière), ${ }^{7}$ Grégoire articule les rapports entre mort et musique, offrant par là-même un cadre sonore du moment du trépas et une spéculation théologique sur la musique eschatologique. Les réflexions sur la mort se trouvent dans divers ouvrages. Le récit le plus direct et le plus engagé, raconté à la première personne, est celui de la mort de sa sœur que
5 Chrysostome, Expositio in Psalmos 41, 1-2; Basile DE CÉSARÉE, Homeliae super psalmos 1, 1; Ambroise, Explanatio Psalmi 1 et 118 , VII, 26-27.

6 Richard RoLLE, Le chant d'amour (Melos Amoris), texte latindel'édition E.J.F.Arnould, introduction et notes par François Vandenbroucke, o.s.b., traduction par les Moniales de Wisques, Paris, Les Éditions du Cerf, 1971.

7 Henri-Irénée MARROU : «Une théologie de la musique chez Grégoire de Nysse», Jacques FonTAINE, Charles KAnNEngiesser (ed.) : Epektasis. Mélanges J. Daniélou, Paris, Beauchesne, 1972, p. 501508. Voir aussi dans le même volume, Eugenio Corsini: "L'harmonie du monde et I'homme microcosme dans le De opificio hominis », p. 455462. En 1970, Jean DANIÈLOU, L'être et le temps chez Grégoire de Nysse, Leiden, Brill, 1970, p. 57-58, avait déjà résumé très brièvement I'utilisation de I'image musicale dans le thème de I'harmonie du monde (repris jusque Hans Boersma, Embodiment and Virtue in Gregory of Nyssa: An Analogical Approach, Oxford, Oxford University Press, 2013, p. 76). À noter également I'article de Barbara FAes de MotToni: "Eventi sonori ed esperienze mistiche in alcuni itinerari teologici tardoantichi e medievali », Eventi sonori nei racconti di viaggio: Antichità e Medioevo, Musica e storia, 13/1, 2005, p. 157-175, qui analyse la présence du son dans I'Exode au départ de la Vie 
de Moïse de Grégoire de Nysse (p. 161-164) et des œuvres de pseudo-Denys, ainsi que le chapitre de Bruce W. Holsinger, Music, Body and Desire in Medieval Culture: from Hildegard of Bingen to Chaucer, Stanford, Stanford University Press, 2001, "'A beautiful, breathing instrument of music': Gregory of Nyssa », p. 46-53. L'auteur, amateur des gender studies qui marquent cet ouvrage, propose un grand nombre des passages musicaux du De opificio hominis analysés dans cette perspective.

8 Grégoire DE NYSSE, Vie de sainte Macrine, introduction, texte critique, traduction, notes et index par Pierre MARAVAL, Sources Chrétiennes 178, Paris, Les Éditions du Cerf, 1971.
Grégoire assiste en ses derniers instants - qu'il suffise ici comme modèle de gamme sonore dans un récit hagiographique, le lecteur en trouvera des centaines jusqu'aux recueils de la vie des saints (Marie Madeleine par exemple prise dans un raptus musical à l'écoute de la musique des anges). Dans la vie de sainte Macrine, ${ }^{8}$ sœur de Grégoire, le rôle de la musique est d'abord de délimiter le temps: non seulement la musique, par son rythme propre, mesure le flux non mesuré du temps qui s'écoule, mais elle rythme les journées et les nuits de l'espace monacal. L'espace-temps de la vie monacale est scandé par le chant des hymnes (cf. Macrine 11) et le mode de vie propulse les religieuses "aux confins de la nature humaine et de la nature incorporelle» (Idem) : I'hagiographie met I'accent sur la transformation opérée par la libération des passions, ces mêmes passions de l'âme dont témoignent les altérations de la voix chez Mersenne. Les religieuses apparaissent dans un corps, elles sont enfermées dans une forme et composées de matière, mais elles ont libéré leur âme des passions aliénantes dont l'impassibilité au moment de la mort sera le signe ultime; ainsi seule la présence du corps les sépare de la vie angélique. Le panégyrique de la vie monacale est évidemment un simple topique chrétien, mais c'est la place de la musique dans son rapport aux passions de l'âme qu'il est intéressant de noter: par le chant quotidien qui rythme les journées et les nuits, les religieuses ne sont plus séparées du chœur des anges que par un cheveu, et le chant continu affirme leur ressemblance avec les puissances incorporelles. Cette place du chant n'est que le prélude à son importance lors du retour final des êtres humains à leur principe. Alors, les deux natures dont I'origine est commune, les anges et les êtres humains, seront réunies en un chœur à I'unisson (cf. In diem. Lum. 9 PG 46, 60 AB) et la métaphore musicale témoigne, de manière traditionnelle, de la perfection de I'harmonie originelle retrouvée. Le moment de passage entre ces moments musicaux - le chant monastique et la réunification eschatologique à l'unisson - est le moment du trépas qui évoque, lui, un monde sonore de la suspension du chant qui fait place à ses deux contraires: le silence et le cri.

La douleur éprouvée à la mort de I'individu est classiquement décrite en termes sonores: les gémissements des proches, de la communauté monastique, voire du pays, le cri de l'univers entier - écho au cri qui précède le dernier souffle du Christ sur la croix (cf. Mt 27, 50). Aux gémissements, larmes sonores et cris de douleur s'oppose le chant surnaturel qui entoure la personne au moment du trépas et qui est signe de la sainteté (et donc consolation pour les présents). De manière symétrique, lorsque Grégoire fait le récit des derniers instants de sa sœur, il rapporte un contraste $d^{\prime}$ affect entre la tristesse de savoir qu'il n'entendrait bientôt plus sa voix et la joie de savoir qu'elle serait unie au chœur des anges (cf. Macrine 22). 
Macrine quant à elle épuise ses dernières paroles en murmures avant d'émettre son dernier souffle (cf. Macrine 23). La voix tend au silence et le murmure est le prélude au cri de douleur des vivants face au silence du mort. Pour dire ses dernières paroles, la voix passe ensuite du murmure au geste: la voix manque, il ne reste plus que le geste des ultimes désirs et les mots insonores ou le mouvement des lèvres qui traduit les derniers élans du cœur (cf. Macrine 25). Thème que reprendra Augustin, sans le contexte dramatique, lorsqu'il affirmera que faire un geste est parler visiblement (cf. De Trinitate, XV, 10, 19). Alors que la force vient à manquer - plus aucun son de la voix - le geste, dans le récit de Grégoire, prend la parole et c'est donc en silence que sont dits les derniers mots. À ce geste des mains répond l'incapacité du geste de Grégoire lorsqu'il doit, quelques instants après cette scène, clore les yeux de sa sœur maintenant morte ( «ma main paralysée par la douleur », Macrine 25).

Au silence des gestes qui remplacent les paroles sonores et au silence qui suit le dernier souffle fait écho I'harmonie naturelle du corps du défunt (qui ne nécessite pas de toilette des morts: signe corporel de sainteté où la nature se dépasse elle-même et ne demande aucun artifice) et le cri des vivants. Grégoire est maintenant face au monde sonore extérieur qui s'était jusqu'alors contenu: contraints, contenus, étouffés de peur du reproche du visage silencieux du mourant, les gémissements des consœurs se font entendre et se transforment à leur tour. "Lorsque la douleur ne put davantage être dominée par le silence, soudain un cri jaillit, déchirant, irrésistible, au point que ma raison ne peut demeurer maîtresse d'elle même » (Macrine 26). Or, cette douleur semble à qui vient de perdre la raison à la fois juste et raisonnable. La passion raisonnable provoque le cri, qui engendre en écho la conscience de l'aliénation de la raison face à la douleur: Grégoire se rend compte de sa propre douleur dans le cri de l'autre. Et ce cri jaillit - «soudain un cri » - : c'est I'irruption irrésistible avec la charge de passion qu'elle contient et libère. Mersenne, sur les traces d'Aristote, cherchera à délimiter précisément le cri, la voix, le bruit, le son, la parole, le chant - chacun porteur d'une nature et d'une sémiologie propres. Grégoire montre combien le cri est parent et témoin de l'irrationnel : il n'articule aucun mot et déchaîne la raison, l'âme se noie lorsqu'elle entend le cri et, emportée par la passion-douleur, elle s'abandonne au flux non mesuré des lamentations.

Grégoire est le maître de chœur, il s'est laissé submerger par le cri et déchirer par la passion que ce dernier manifeste, mais il récupère sa raison, comprend la déraison du cri et du désordre causé par les lamentations (cf. Macrine 27). Le chant funèbre n'est pas ici harmonie consolatrice ou écho de I'harmonie céleste ayant accueilli I'âme du défunt et il doit se 
changer en psalmodie unanime (cf. Idem) : " on passa donc la nuit autour d'elle à chanter des hymnes, comme pour le panégyrique des martyrs » (Macrine 33). Et lorsque le lendemain matin, la foule $d$ 'hommes et de femmes arrive pour rendre hommage et accompagner le cortège funèbre, Grégoire s'occupe à nouveau des règles du chant: "Je fis en sorte que la psalmodie en provenance des uns et des autres soit unique, bien rythmée et harmonieuse, comme dans le chant d'un chœur, parfaitement homogène grâce à la mélodie commune à tous » (Idem). De la même manière, Grégoire commente la musique qui accompagne le convoi funèbre: "du commencement jusqu'à la fin la psalmodie était chantée d'une seule voix ». Mais cette harmonie - composition des présents, homogénéité musicale - ne dure que le temps de la marche rythmée du convoi. Une fois le cortège entré dans l'église, "lors d'une pause dans la psalmodie ", les consœurs de la défunte tournent leur regard vers le visage de cette dernière et commencent à crier sans mesure : le silence musical ouvre le temps au regard. Et le regard provoque à nouveau le cri : irrationnel, sans mesure, témoin de la conscience de la perte. L'interruption musicale permet l'irruption a-musicale car irrationnelle du cri. La rationalité du nombre musical est rompue :

L'une d'elles se mit à crier sans mesure [...]. Il s'en suivit une confusion inconsidérée, qui rompit le bon ordre et le caractère sacré de cette psalmodie, car tous éclataient en sanglots [...]. C'est avec peine que par signe nous demandâmes le silence; le chantre invita alors à la prière en entonnant les oraisons habituelles de l'église, et le peuple revint aux dispositions de la prière (Macrine 34).

Le chant dispose les individus et s'oppose à la confusion. Le pouvoir de la musique est réaffirmé, la psalmodie pacifie les âmes et se pose en contraste avec les passions qui s'expriment de manière irrationnelle par I'irruption du cri et l'éclatement des larmes. Le bruit (cri, sanglots) s'oppose au chant qui agit sur l'assemblée et annonce les hymnes célestes, comme le désordre du cri à l'ordre de la psalmodie et la confusion des sanglots à I'harmonie des oraisons.

Pour dépeindre la mort, la voix use de toutes ses nuances: le tableau sonore mortuaire, encadré par le chant, qu'offre le récit de Grégoire montre une palette du silence au cri. La voix dessine les passions contrastantes : régularité de la vie monastique rythmée par la pratique quotidienne du chant, caractère exceptionnel et donc irrégularité de la mort qui rompt le chant. Le chant quotidien prend fin au jour ultime, la voix devient murmure avant de laisser place aux paroles silencieuses des gestes et au der- 
nier souffle. Le cri et le bruit des sanglots des vivants en ce même jour rompent les chants du chantre et des chœurs - avant que le tout ne se résolve dans I'eschatologie musicale. Grégoire, par son art rhétorique, dépeint les différentes tonalités du moment de la mort évoquant I'univers sonore qui devient le miroir des passions. Si les images ont leur place (I'ultime geste des mains, I'harmonie du corps, les yeux clos, les cierges du convoi), les images sonores sont tout aussi, sinon plus importantes car le son, du bruit au chant, est miroir des passions et donc de l'intériorité des protagonistes. Le contraste n'est pas tant entre l'immobilité du corps mort et le mouvement du vivant - la main de Grégoire se paralyse devant le corps de sa sœur à peine morte - mais entre le silence du premier et le hurlement du second. Ce double contraste sera rendu de manière aussi dramatique que le récit de Grégoire dans le groupe de terre cuite sculpté de la lamentation sur le Christ mort (1460) conservé à l'église de Santa Maria della vita à Bologne: le corps étendu immobile est encerclé de figures contorsionnées en larmes et de figures hurlantes au mouvement et au cri déchirant - «soudain un cri ».

\section{Sermon De voce et verbo}

Parmi les spéculations sur la voix, il est un lieu où celles-ci se font plus intenses et denses de contenu théologique: il s'agit des sermons ou commentaires sur l'évangile de Jean et en particulier sur l'affirmation de JeanBaptiste qui se définit comme étant une voix. Augustin y consacre diverses pages, notamment dans ses sermons 288 et 289. On pourrait y ajouter les textes augustiniens des spéculations sur I'Incarnation dans lesquelles Dieu s'est fait simple son éphémère - il assume un corps, le Verbe devient voix, son insignifiant dans son impermanence. Pour Augustin, Jean-Baptiste est celui qui distingue intérieurement le Créateur enveloppé dans la figure du nouveau-né. Jean voit intérieurement la divinité du Christ et I'annonce. II est I'homme capable de voir Dieu en I'homme et le montre du doigt (cf. sermon 288, 1). À la vision intérieure suit donc le geste. Simultanément au geste - mille fois transcrit dans l'iconographie chrétienne, le geste de la main seul suffit à identifier Jean-Baptiste quand bien même tous ses autres attributs, vestimentaires par exemple, sont absents - se donne la parole (absente des récits iconographiques, sauf à ajouter un phylactère à la figure). Cette première parole dit Dieu dans I'homme ( Voici I'Agneau de Dieu », Jean 3,29). Et Jean-Baptiste aura à rendre compte de cette parole, ainsi les envoyés lui demandent: "Qui es-tu? ». Question à laquelle Jean répond "Je suis la voix de Celui qui crie dans le désert» (Jean 1, 23). 
9 Quid est vox? quid est verbum? Quid? Audite quod in vobis ipsis approbetis, et vobis ipsis a vobismetipsis interrogati respondeatis.

10 Verbum, si non habeat rationem significantem, verbum non dicitur. Vox autem, etsi tantummodo sonet, et irrationabiliter perstrepat, tamquam sonus clamantis, non loquentis, vox dici potest, verbum dici non potest.

${ }^{11}$ Nescio quis ingemuit, vox est: eiulavit, vox est. Informis quidam sonus est, gestans vel inferens strepitum auribus sine aliqua ratione intellectus.
Augustin, qui résume l'identité de Jean à une voix « Jean est une voix » (sermon 288, 2), commente cette réponse en disant que la voix précède le verbe comme Jean le Christ (bien que le Fils-Verbe soit coéternel au Père et donc précède Jean). Si I'on doit considérer la voix et le verbe tous deux au bord du Jourdain, il faut définir l'un et I'autre: "Qu'est-ce que la voix? Qu'est-ce que la parole? » (cf. sermon 288, 3). Et de manière toute augustinienne, I'individu trouve la réponse à ces questions en lui-même par l'introspection ( Écoutez ce dont vous allez reconnaître la vérité en vous-mêmes, en vous interrogeant et en vous répondant intérieurement ", Idem). ${ }^{9}$ Cette dernière mène à des considérations d'ordre sémiologique et établit la distinction entre le son insignifiant et le son signifiant, entre le son chargé d'une signification (la parole) et le son dépourvu de sens (le bruit).

\section{Le cri insensé}

Pour Augustin - mais il pense différemment dans les Enarrationes in Psalmos par exemple -, le son inarticulé n'a pas de sens. Seule la parole est pourvue d'une signification. Le cri, dépourvu de parole, est réduit à un bruit, "un bruit qui n'a pas de sens » (sermon 288, 3) n $^{10}$ - comme si les passions de l'âme traduites par le son non articulé - du cri au soupir n'avaient en soi aucune signification, ce qu'Augustin lui-même contredit, voire renverse. En effet, dans les Enarrationes in Psalmos, les paroles ne peuvent traduire les passions de I'âme. Ces dernières dépassent la raison - douleurs et joies incommensurables et donc intraduisibles en mots par définition mesurés - et sont traduites par le son de la voix qui se libère du carcan des voyelles et des syllabes. Alors, le chant qui exprimait la joie abandonne les mots et, affranchi des limites imposées par les paroles, il se libère, se répand, se dilue et se condense dans le son (cf. Enarrationes in Psamos 32 II discours 1, 8). Reprenant la vieille définition d'Aristote, dans les sermons Augustin insiste au contraire sur la distinction entre voix articulée (parole) comme véhicule de sens et voix non articulée (bruit) privée de sens. Dans le cri « on peut dire qu'il y a voix, mais il n'y a point parole. Un gémissement est une voix; un cri plaintif est une voix. La voix est comme un son informe qui retentit aux oreilles sans rien dire à l'entendement » (sermon 288, 3 ; cf. sermon 289, 3). ${ }^{11}$ La parole, elle, s'adresse à l'intelligence par l'intermédiaire des oreilles. Elle passe par les sens et, en passant, elle dépose sa signification dans I'oreille interne de I'entendement: parler est signifier, apporter du sens, permettre d'apprendre. Or si "un cri jeté c'est une voix », Jean n'est qu'une voix jetée dans le désert, un cri insensé - «Je suis une voix qui crie dans le désert». 


\section{La matière de l'idée}

Une autre supériorité de la parole sur la voix est son efficacité indépendante de son émission sonore: "séparée même de la voix, la parole peut avoir son efficacité ; tandis que sans la parole la voix est vaine » (sermon 288, 3). La parole, idée et raison, est une pensée présente dans l'esprit et conservée dans la mémoire. Conçue par les facultés supérieures de l'âme, elle est déjà entièrement formée, il ne lui manque que la matière, la voix, pour être entendue par l'autre. "C'est une idée [...] que conçoit I'intelligence et qui se prépare à s'en échapper pour s'insinuer dans l'esprit de I'auditeur» (Idem). Elle n'est pas encore communiquée, et en cela elle n'a pas encore de relation à l'autre, mais elle est aussi, par là-même, libérée de toute langue: "ce que tu veux dire n'est encore formulé dans aucune langue» (Idem), bien qu'il soit formé dans l'esprit. L'intériorité de I'individu est alors l'espace pré-babylonien, où la forme de l'idée se donne sans I'intermédiaire de la langue, mais cette pensée insonore n'a qu'une consistance narcissique car elle ne peut être communiquée si elle ne s'incarne pas dans la matière, forcément particulière, de la langue sonore (grecque, latine, punique, hébraïque, cf. Idem). Si le corps est règne de la division, la voix divise l'unité de la pensée en langage particulier. La pensée s'échappe de I'unité intérieure pour s'insinuer dans l'esprit de l'autre par I'intermédiaire des corps (voix-oreille). "Supprimer ces différents interlocuteurs, et la parole intérieure n'est ni grecque, ni latine, ni punique, ni d'aucune autre langue. Elle a besoin, pour se montrer, d'un son de voix connu de celui à qui on veut I'adresser » (Idem). ${ }^{12}$ L'idée dans I'esprit transcende la lettre, le mot, la voix. Le son de la voix dit la division, des sons, des lettres et des différentes langues des interlocuteurs qui ne se comprennent pas I'un I'autre.

Enfin, l'idée perdure en celui qui la prononce tout en arrivant à celui qui I'écoute. La troisième réflexion augustinienne sur la voix traite de cette dernière comme intermédiaire - matière et media - de la pensée intérieure. L'idée (pensée, conception de l'âme, parole) recourt au ministère de la voix, la première étant engendrée avant la seconde - «la parole existe avant de paraître sous forme de voix» (Idem) - . Précédence qui se renverse dans le chef de l'auditeur, ce dernier connaissant d'abord la voix et ensuite la pensée: "la parole est en moi antérieure à la voix [... . En toi au contraire, c'est I'oreille qui est frappée d'abord du son de ma voix pour porter ma pensée, ma parole à ton esprit » (Ibid., 4). ${ }^{13} C^{\prime}$ est pourquoi, puisque le Fils est parole, pensée, verbe généré dans I'intériorité du Père, il est antérieur à Jean qui est voix, quand bien même Jean comme voix qui transmet la parole est antérieur au Christ.
12 Remove diversitatem auditorum, et verbum illud, quod corde conceptum est, nec graecum est, nec latinum, nec punicum, nec cuiusquam linguae. Talem vocem quaerit procedendi, qualis assistit auditor.

13 Praecessit ergo verbum vocem meam, et in me prius est verbum, posterior vox: ad te autem, ut intellegas, prior venit vox auri tuae ut verbum insinuetur menti tuae. 
14 Ideo proprie dictus est vox, tamquam omnium vocum signaculum atque mysterium.
L'enquête sur les mécanismes du langage et de I'audition, sur les lieux et les temps du son de la voix et de la pensée guide la compréhension de questions théologiques.

\section{La voix et les voix}

Ces questions théologiques expliquées par la différenciation entre matière de la voix et forme de la pensée conduisent également à approfondir les desseins divins dont elles témoignent. Ce qui mène Augustin à distinguer la voix (Jean-Baptiste) et les voix (prédicateurs, âmes pieuses et tout ce qui, d'une manière ou d'une autre, prêche le Verbe). "La voix se personnifiait dans saint Jean, mais seul il n'était pas la voix » (sermon 288, 4). Ainsi, le Verbe conçu par et dans $n^{\prime}$ importe quelle intelligence humaine produit et génère des voix avant son incarnation: "autant de voix qu'il a fait entendre sans sortir du sein de son Père; mais après toutes ces voix le Verbe est venu lui-même et tout seul, porté par sa chair, comme par sa voix » (Idem). Les patriarches, prophètes ou sibylles sont autant de voix qui profèrent le Verbe avant la venue du Verbe dans la voix et dans la chair - dans la chair de la voix et dans la voix de la chair (I'Incarnation). Si les voix étaient générées par le Verbe avant qu'il ne soit voix, elles se sont également toutes rassemblées dans la personne de Jean - une voix. Alors Jean, une voix, devient annonciation et personnification du Verbe, I'incarnation de toutes les voix avant I'Incarnation du Verbe. "Si donc il a été le seul et spécialement appelé la Voix, c'est qu'il était comme le symbole et la représentation de toutes ces autres voix » (Idem). ${ }^{14} \mathrm{~L}^{\prime}$ identité de Jean est d'être une voix, la voix de l'Autre qu'il a intérieurement perçue et reconnue, une voix qui rassemble toutes les voix précédentes et qui opère le passage entre toutes les voix et I'unique voix du verbe incarné. Jean est la Voix en ce qu'il unifie les diverses voix prophétiques - en cela il dit la fin des patriarches - et en ce qu'il annonce la voix unique - il dit le début, I'arrivée, I'avènement du Fils. La Voix est alors le pont entre la génération des anciens et le Verbe qui se fait corps, chair, son, voix : "il voulut que son corps sacré fût en quelque sorte sa voix spéciale » (sermon 288, 5). Dans I'exégèse augustinienne au verset évangélique du sermon 288 (et en partie du sermon 289), tout se joue dans les modulations de la voix: le père de Jean-Baptiste a perdu l'usage de la parole, il est devenu muet car il n'a pas cru que la naissance de son fils serait possible. Jean-Baptiste, comblant le mutisme du père, prononce une parole qui résume à elle seule l'essence du message chrétien ("voici l'Agneau »), alors que lui-même est un cri (aux antipodes du silence muet), une voix 
qui crie dans le désert, la Voix personnification de toutes les voix, une voix qui prépare la voie au Fils.

\section{Le corps de la voix chez Jean Scot}

Dans son Commentaire sur l'évangile de Jean, Jean Scot offre une spéculation sur la voix qui oriente l'iconologie de la voix vers une véritable icône de I'Autre. Si pour Mersenne sur les traces augustiniennes, la voix est le miroir de I'âme (cf. Traitez de la voix, p. 8), pour Jean Scot la voix est I'incarnation de I'autre et le dépassement de soi. Aux questions "Qui es-tu? » et "Que dis-tu de toi-même? » posées à Jean-Baptiste, ce dernier répond « Je suis la voix de celui qui crie dans le désert » (In Iohannis Evangelium Commentarius, I, 27 [303D]; cf. Jean 1, 23). ${ }^{15}$ L'identité de Jean se résume dans sa voix, il ne répond ni «Je suis Jean », ni même "Je suis un homme », mais il se définit par sa voix. Cette première identification entre la voix et la personne ne dit cependant pas tant I'identité que I'altérité. En effet, Jean ne dit pas "Je suis ma voix», mais "Je suis la voix de celui qui crie dans le désert », voire simplement "Je suis une voix».

"Si vous me demandez, dit-il, ce que je dis de moi-même, sachez que je suis une voix» (Idem. "Si quaeritis, inquit, quid de meipso profero, cognoscite quia ego sum vox»). "Ego sum vox» (Ibid., [304A]) est la réponse au "Qui es-tu? », car Jean se dépouille de lui-même pour n'être plus que voix et pour être au-delà de lui-même précurseur du Verbe. Jean est la voix dans le désert qui annonce le Christ : il a abandonné sa condition humaine.

Il a laissé derrière lui toutes les réalités qui sont contenues dans les limites de ce monde, il s'est élevé dans les hauteurs, il est devenu la voix du Verbe [... $]$. Je suis donc une voix, dit-il, non pas ma voix, mais la voix de celui qui crie (Idem). ${ }^{16}$

Si, pour l'éthique musicale néoplatonicienne et chrétienne classique, I'individu devient ce qu'il écoute, les mélodies vocales ou instrumentales modelant I'âme, pour Jean Scot dans ce commentaire à Jean, la voix transcende l'individu et dit ce qu'il n'est pas. Jean n'est pas un prophète (cf. Ibid., [303C]), il n'est pas non plus simplement lui-même, il est la voix d'un autre, de I'Autre incarné. La voix ne révèle plus I'âme - ses passions, ses idées - , mais elle est la révélation de Dieu. Jean est déjà divinisé car il a laissé les réalités contenues dans les limites du monde. La voix n'est plus la limite des mots dont il faut se libérer pour pouvoir dire Dieu, elle
15 «Quis es?, Quid dicis de teipso? " «go sum vox clamantis in deserto ». Jean Scot, Commentaire sur l'évangile de Jean, Edouard Jeauneau (éd.), Sources Chrétiennes 180, Paris, Les Éditions du Cerf, 1972. Voir aussi Augustin, In Iohannis evangelium IV, 7. La pensée augustinienne dans I'œuvre de Jean Scot est étudiée depuis longtemps: Brian Sтоск: «Observations on the use of Augustine by Johannes Scottus Eriugena ", Harvard Theological Review, 1967, p. 213-220; Giulio BonAfEde: «Presencia de san Agustin en Escoto Eriugena ", Augustinus, 16, 1980, p. 263-285; Thomas HALTON (ed.), Studies in Augustine and Eriugena, Washington, The Catholic University of America Press, 1992. En revanche, les aspects musicaux de Jean Scot sontpeuétudiés. Danslesannées 1920, un article résume sa pensée musicale: Jacques HANDSCHIN : "Die Musikanschauung des Johannes Scotus Erigena », Deutsche Vierteljahresschrift für Literaturwissenschaft und Geistesgeschichte $\mathrm{V}$, 1927, p. 316-341. Une autre contribution déjà ancienne indique qu'il ne faut pas voir chez Jean Scot autre chose que des références traditionnelles aux théories musicales classiques proposées dans les commentaires du Timée: Ernst L. Waeltner, Organicum melos: Zur Musikanschauung des Iohannes Scottus Eriugena, Munich, Bayerische Akademie der Wissenschaften, 1977. Voir également Marie-Elisabeth 
Duchez: «Jean Scot Erigène premier lecteur du De institutione musica de Boèce?", Werner Beierwaltes (ed.): Eriugena: Studien zu seinen Quellen, Heidelberg, Winter, 1980, p. 165-187. Toujours sur la réception de Boèce, voir Klaus W. Niemölter: "Die Musik im Weltbild des Johannes Scotus Eriugena », Frank Hentschel (ed.): Musik, und die Geschichte der Philosophie und Naturwissenschaften im Mittelalter: Fragen zur Wechselwirkung von musica und philosophia im Mittelalter, Leiden, Brill, 1998, p. $293-$ 304 et Raymond ERICKSON: "Eriugena, Boethius, and the Neoplatonism of Musica and Scolica enchiriadis", Nancy Kovaleff Baker (ed.): Musical humanism and its legacy: Essays in honor of Claude V. Palisca, New York, Pendragon Press, 1992, p. 53-78. Jacques VIRET, «Harmonie musicale et harmonie naturelle à l'époque carolingienne: Exégèse d'un passage de Jean Scot Erigène », Danielle Buschinger, André CRÉpIn (ed.): Musique, littérature et société au Moyen Âge, Paris, Champion, 1980, p. 8598 présente la musique comme réduction de la création à travers quelques passages du $P e-$ riphyseon. Sur ce thème, voir également le chapitre de Cecilia PANTI: "Giovanni Scoto: Razionalità ed estetica dell'armonia universale », dans son Filosofia della musica: Tarda antichità e medioevo, Rome, Carocci, 2008, p. 128-138 et Gabriela I. CURRIE: «Concentum caeli contient le Verbe que rien ne peut contenir. De manière symétrique, Jean n'est plus lui-même, il ne connaît plus ni «mien» ni «moi » (cf. Basile de Cesarée, Homeliae in Hexameron, X, 7). ${ }^{17}$ II n'est plus rien qui lui soit propre, mais il est toute connaissance relative: il est la voix de l'absolu. "Jean est donc la voix du Verbe, du Verbe qui crie, c'est-à-dire du Verbe qui prêche par sa chair» (In Iohannis Evangelium Commentarius, I, 27 [304A]). Le corps du Verbe est la voix de Jean devenue la voix de I'Autre.

Une voix qui crie en Israël réduite au désert, car vouée à la lettre de la loi et vidée de son esprit. Une voix qui crie en toute terre consumée dans la lettre vide de sens. "C'est dans cette solitude que la voix du Verbe et le Verbe lui-même se sont fait entendre » (Ibid., [304B]). La voix porte alors l'esprit là où la lettre règne et provoque de ce fait un souffle nouveau: elle provoque. Ce souffle nouveau envahit la solitude du désert et y apporte un sens nouveau: elle transcende doublement la lettre car elle y inclut le sens de l'esprit et I'incarne dans la chair de la voix. Jean Scot reprend le vieux thème de la voix matérielle et sensible comme instrument de l'âme: "La voix est I'interprète de l'esprit» (Idem). Ce passage entre I'invisible et l'audible, entre les pensées silencieuses et l'âme invisible d'une part et la voix, invisible mais audible, matérielle et médiation entre l'intériorité de celui qui pense et I'extériorité (I'auditeur), d'autre part, ce processus de communication est décrit par la plupart des Pères grecs et latins. Ce que l'esprit a ordonné de manière invisible, il le confie ensuite à la voix pour le faire parvenir sensiblement aux auditeurs.

Jean est image sonore du Verbe. Une image qui diffère néanmoins de I'image qu'est le Fils par rapport au Père. Augustin soulignait comme propriété du Verbe son identité avec le Père bien qu'il ait besoin du Père pour être. "La forme dans I'Image » est I'image du Père dans le Fils, parfait en terme de beauté et proportion, de première égalité et en rien discordant au Père, identique à ce dont il est I'image (cf. "species in imagine ", De Trinitate, VI, X, 11). Ce Verbe, pour Scot, devient I'esprit de toutes choses que manifeste la voix (cf. In Iohannis Evangelium Commentarius, I, $27[304 C]$ ). Et pourtant, le désert est aussi I'altérité radicale de la nature divine, différente de toutes choses et donc indicible et incommunicable. "Celle-ci [la nature divine], en effet, est désertée par toute créature, puisqu'elle surpasse toute intelligence, alors qu'elle-même ne déserte aucune intelligence » (Idem). Nature présente en toutes choses alors qu'elle $n^{\prime}$ est contenue par aucune, sa hauteur l'éloigne de toute comparaison, raison, parole, métaphore. "C'est donc dans cette divine hauteur que crie le Verbe » (Idem). Et ce dernier se dédouble alors en création et incarnation. Incarnation en la voix du Fils, Création lorsque "Dieu dit» car alors «Dieu» indique le Père, «dit» indique le Verbe (cf. Ibid., [304D]). 
Son cri est la création de toutes les natures. C'est lui qui appelle les choses qui sont comme celles qui ne sont pas, c'est par lui que Dieu le Père a crié, c'està-dire, a créé tout ce qu'il a voulu créer. Il a crié de manière invisible, avant que le monde fût fait, pour que le monde soit fait; il a crié en venant dans le monde de manière visible, pour que le monde soit sauvé (Ibid., [305A]). ${ }^{18}$

La théologie de la voix, mutée en théologie du cri (clamor), inclut de cette sorte une double voix: d'une part une voix invisible, indicible et créatrice dont la nature transcendante échappe à l'entendement rationnel et donc au langage et, d'autre part, une voix de chair dans l'incarnation. Le jeu sur «crié » et «créé » (clamavit id est creavit) souligne dans I'allitération la nature performative du cri de Dieu: pour lui, crier c'est créer comme penser, dire et faire sont un. ${ }^{19}$ Le premier cri du Verbe, dans la solitude du désert de sa totale altérité, est le cri créateur qui déchire I'éternité et pose toutes choses en devenir. Le second cri du Verbe est son incarnation conçue comme salut de ce même monde déchu auquel la voix redonne l'éternité. La voix comprend ainsi le mystère de la création, I'annonce de l'incarnation par la figure de Jean et le plan du salut: de la création à la rédemption, la voix indique la dépendance du monde matériel au monde divin et les manifestations de ce dernier. Elle indique le Père créateur et son cri dans la lointaine solitude de sa transcendance, le précurseur du Fils qui transcende son «moi» («Qui es-tu? « "Je suis une voix »), et le Fils même dans le cri rédempteur de son incarnation (jusqu'au dernier cri sur la croix, cf. Mt 27, 50). Le cri, la clameur, la voix du Verbe dessinent dans le Commentaire à l'évangile de Jean de Jean Scot le contour sonore des extrémités de la rédemption (création et salut / Père et créatures).

\section{La voix comme miroir de l'âme chez Mersenne}

Platon a creu que la voix monstre l'interieur des hommes [...] \& disoit: parle, afin que ie te voye (Traitez de la voix et des chants, p. 10). ${ }^{20}$

Mersenne est lui aussi, comme Augustin, Grégoire de Nysse et Jean Scot, I'héritier de la philosophie platonicienne et néoplatonicienne à laquelle il ajoute les problèmes aristotéliciens, notamment dans ses discussions sur la voix. Il n'est pas ici question d'analyser de manière exhaustive la richesse des spéculations sur la voix chez Mersenne, mais d'en donner des aperçus dans le cadre d'une iconologie de la voix qui, au xvirème siècle, migre du discours théologique au discours musical, médical et anatomique. Si les quis dormire faciet? Eriugenian cosmic song and Carolingian planetary astronomy », David Butler Cannata et al. (ed.): Quomodo cantabimus canticum? Studies in honor of Edward H. Roesner, Middletown, AIM, 2008, p. 15-35.

${ }^{16}$ Itaque ego sum vox, non mea vox, sed clamantis vox.

17 Sur la distinction entre le «mien » et le «moi », voir cette homélie attribuée à Grégoire DE NySSE ou Basile de CÉsARÉE [?], Sur l'origine de l'homme (Homélies X et XI de I'Hexaéméron), Alexis SMETS, Michel van Esbroeck (ed.), Sources Chrétiennes 160, Paris, Les Éditions du Cerf, 1970.

18 Clamor eius naturarum omnium conditio est. Ipse enim vocat ea quae sunt tanquam quae non sunt, quia per ipsum deus pater clamavit, id est creavit cuncta quae fieri volvit. Clamavit ille invisibiliter, priusquam fieret mundus, mundum salvari. Prius clamavit aeternaliter per solam suam divinitatem ante incarnationem; clamavit postea per suam carnem.

${ }^{19}$ Sur I'origine de la parole éternelle comme cri, cf. Periphyseon, II, 24.

20 Sur cet auteur, voir par exemple les récentes contributions de Brigitte VAN WYMEeRSCH: «Marin Mersenne et les rapports texte-musique », Anne-Madeleine GouLET, Laura NAudeix (ed.): La fabrique des paroles de musique en France à I'âge classique, Wavre, Mardaga, 2010, p. 57-75; "Proportion, harmonie et beauté 
chez Mersenne. Entre lecture analogique et lecture physicomathématique de lamusique », Sabine Rommevaux, Philippe Vendrix, Vasco Zara (ed.) : Proportions, Turnhout, Brepols, 2012, p. 249-262. Surlepassage entre lamusique comme reflet du Beau et la musique comme expression de I'âme dans la perspective esthétique du Xvirème siècle, voir Dean T. MAcE : «Marin Mersenne on language and music ", Journal of Music Theory, 14/1, 1970, p. 2-34.

21 Sur le thème, mille fois étudié tant en histoire de l'art qu'en musicologie, de la mimesi (des réflexions de Platon et Aristote aux modulations du concept dans l'esthétique et la théorie des beaux arts du xvirème siècle), voir par exemple l'ouvrage récent La musique face au système des arts ou les vicissitudes de l'imitation au siècle des Lumières, MariePauline MARTin, Chiara SAVETTIERI (ed.), Paris, Vrin, 2013. traités de la seconde moitié du Xvirème siècle déplacent l'accent de l'écoute intérieure (philosophique et théologique) à la mécanique de l'oreille externe (anatomique et médicale), Marin Mersenne montre combien voix et intériorité sont encore indissociablement liées malgré I'intérêt du père Minime pour I'anatomie de I'appareil phonétique. L'envoi du Traitez de la voix et des chants - "A Monsieur Halle seigneur de Boucqueval etc. » - révèle le fondement religieux qui anime le philosophe de la musique et père de I'ordre des Minimes: Dieu a donné la Musique aux êtres humains et Platon, le divin philosophe, "s'en est tousiours servy pour exprimer ses pensees ». Si David est le père des thérapeutes musicaux, se servant de sa lyre et de ses chants pour soigner Saül, Platon est le père des philosophes de la musique. Le cadre que pose Mersenne dans cet envoi le montre comme héritier direct du néoplatonisme chrétien - si pour Grégoire la musique est éveil du désir de l'Éternité, Mersenne affirme encore:

Ie sçay que c'est là où tous vos desirs sont portez, \& que I'Harmonie Archetype vous touche davantage que I'Elementaire, dont nous usons maintenant, laquelle n'est que I'image, ou, I'ombre de la Divine.

Mersenne confirme si besoin est la suprématie de la musique supraterrestre sur la musique sensible clairement établie par Augustin. Le chant et tous les instruments de musique ne sont que des ombres et de pâles reflets de la musique archétypale. La musique sensible est donc d'abord et avant tout appauvrissement: elle est simple imitation de I'harmonie céleste et divine avant d'être imitation et représentation des passions de I'âme humaine. Imitation des passions de I'âme, la musique est, comme la voix, interface entre intériorité et extériorité: elle projette les mouvements de l'âme sur la scène sonore mais, à la différence de la voix, elle peut imiter des passions contraires à celles qui se meuvent dans l'âme du chanteur. ${ }^{21}$ La distance entre la voix et le chant est alors celle entre la nature et I'artifice.

Il n'est pas nécessaire que nous exprimions nos propres mouuemens, ou passions, il suffit que nous imitions les passions des autres, ou du sujet proposé, comme il arrive presque toujours à ceux qui chantent pour donner du plaisir aux auditeurs, car encore qu'ils soient tristes, ils peuvent chanter des Airs fors gays, ou des Airs tristes, encore qu'il soient pleins d'alegresse (Traitez des chants, p. 93).

L'artiste s'efface devant le sujet qu'il incarne, comme Jean devant le Verbe. La fonction de la musique et le rôle du chanteur est d'exprimer les passions de l'âme pour le bon plaisir des auditeurs (ce qui inclut que le 
plaisir peut venir d'un air triste). À la différence de la voix, la voix chantée et mise au service de la composition donne corps aux passions des autres: dans I'air, la voix n'est pas le reflet matériel du chanteur, mais le miroir de I'autre. Cette représentation des passions conserve son antique lien à la catharsis grecque, et le théâtre sonore sert l'éthique plus encore que le pouvoir des images:

C'est pourquoy les airs peuuent representer les divers mouuemens de la mer, des cieux, \& des autres choses de ce monde, d'autant qu'on peut garder les mesmes raisons dans les intervalles de la Musique qui se rencontrent aux mouuemens de I'ame, du corps, des Elemens, \& des cieux. De là vient que la Musique sert plus à la vie Morale, \& est plus propre pour les moeurs que la peinture, laquelle est comme morte, mais la Musique est vivante, \& transporte en quelque façon la vie, I'ame, I'esprit \& I'affection du Chantre, ou du Musicien, aux oreilles \& dans I'ame des auditeurs (Traitez des chants, p. 92).

Le musicien, comme le peintre, peut représenter n'importe quel élément visible (la mer, le ciel et les autres choses de ce monde). Il est capable de dépeindre grâce à la matière propre de son art toutes les réalités matérielles - non seulement la mer, mais le mouvement de la mer - et psychologiques. Mais, à la différence du peintre, le musicien représente en mouvement les mouvements du corps, de l'âme et du monde élémentaire toujours soumis au temps et donc aux mouvements et aux altérations. D'où I'idée selon laquelle la peinture représente la nature morte, la musique la nature vivante; la peinture fige la nature vivante, la musique «transporte» la nature vivante - «la vie» - dans l'âme et le corps (les oreilles) de I'auditeur. C'est pourquoi l'Église «s'est servie de la Musique afin de transporter les esprits des fideles iusques au ciel » (Idem). L'antique thème du pouvoir de la musique est réaffirmé : I'art des sons imite les passions de I'âme et, à travers le corps, les fait pénétrer dans l'âme. Ce n'est pas la faculté de représentation qui manque à la peinture, mais ce double transport: la musique transporte les passions par le mouvement du son et les imprime dans les mouvements de l'âme. Ce transport pose également le corps au centre de son action: la vue perçoit I'objet, I'oreille le reçoit. Entre la perception de la vue et la réception de l'ouïe, il y a la distance entre l'impression de l'image et la pénétration du son.

Une question posée par Mersenne à la suite de ces considérations participe de l'iconographie de la voix en interrogeant les frontières mobiles et floues entre voix et chant. "Quand, \& à quel moment le son, ou la voix commence à estre chant »? ( Traitez des chants, p. 93). Écho à la question d'Augustin au livre XII des Confessions lorsqu'il se demande si I'on peut distinguer le son du chant (Confessions, XII, 29, 40). Peut-on, demande 
22 Sur l'affinité entre musique et rhétorique et sur les recommandations de Mersenne pour illustrer musicalement efficacement les passions, voir David H. Duncan : "Persuading the affections: Rhetorical Theory and Mersenne's advice to harmonic orators ", Georgia CowART (ed.) : French musical thought, 1600-1800, Ann Arbor, UMI, 1989, p. 149-176.
Mersenne, déterminer de manière catégorique quand la voix n'est plus simple parler mais devient chant? Cette question, il la pose de manière philosophique, détachée d'un contexte précis, d'une supposée positivité d'un morceau musical. Mersenne n'est pas ici un musicologue des partitions: le philosophe de la musique questionne la matière et la nature de la musique.

$S^{\prime}$ il est très difficile de remarquer le commencement du mouvement, \& du temps, \& par consequent celuy du son, qui n'est autre chose qu'un mouvement, il n'est ce semble pas moins difficile de determiner quand le son commence d'estre Chant (Traitez des chants, p. 93).

D'autant plus que même sans altération, le son peut être dit chant, bien que le chant parfait "désire des changements de son» (Idem, p. 94) car la perfection demande harmonie, et donc mélange et diversité (le plaisir naît de la variété - cf. Traitez des chants, p. 176). La question s'est déplacée du sens au son: il s'agit moins de la distinction entre voix (bruit insensé) et parole (son articulé) que de la frontière entre parole et chant. Le son continu (bourdon, "un simple son qui tient ferme»), le discours des orateurs («qui font des intervalles sensibles») et des prédicateurs («qui chantent en parlant») sont autant de cas limites entre parler et chanter, mais qui tous appartiennent à la catégorie du chant. ${ }^{22}$ Or, si ce chant est travestissement de la voix par l'artifice - le chanteur projette des douleurs et des joies qui ne lui sont pas propres, il s'identifie pour un instant à la gamme d'affects que son art reproduit - la voix naturelle, elle, qu'elle soit cri ou parole, reflète dans la matière sonore ce qui habite l'intériorité de l'individu. Ce qui mène Mersenne à émettre I'hypothèse selon laquelle, s'il est possible de connaître le tempérament et les passions de l'âme par les traits du visage (physionomie) et par les lignes de la main (chiromancie), il est également possible de les connaître par la voix (plus encore que par la parole ou le rire) (cf. Traitez de la voix, p. 61). Mersenne réduit les qualités de la voix à trois grandes catégories: faible et forte, claire et rauque, grave et aiguë (Traitez de la voix, p. 6). La voix forte et robuste est I'indice d'un tempérament chaud, bien que parfois I'observation contredise cela - la «phoniscopie» n'est pas sans faille. Pareillement la définition des déformations de la voix est relative: "ce que I'un croit estre vice \& imperfection dans la voix, I'autre l'estimera peut estre une perfection » ( Traitez de la voix, p. 43).

Une voix âpre, aigre et rude - tel un corps brut et «raboteux »blesse l'ouïe et c'est là son vice, son défaut, son imperfection reconduite à ses causes physiologiques et suivie des remèdes (cf. Idem; Ibid. p. 
44-46). La voix âpre, la voix courte et tremblante, la voix dure et rigide, la voix cassée, étouffée et ténébreuse, la voix rauque causée par les frayeurs ou les veilles excessives sont autant d'altérations de la voix reconduites à des altérations de la psyché (crainte et frayeur pour la voix rauque) et des organes phonétiques (les muscles trop faibles qui tremblent en remuant les cartilages de l'artère pour la voix tremblante, la quantité de pituite et d'autres humeurs qui obstruent les organes vocaux responsables de la voix ténébreuse etc.). La liste des remèdes pour conserver ou guérir les altérations (vices et imperfections) de la voix comprend tant des soins moraux que médicaux (exercice physique, chant et abstinence des plaisirs immodérés pour conserver la voix, poireaux et oignons, graines de choux broyées et sucrées, tisane d’orge et de réglisse, décoction de figues, sirop de violette et de nénuphar pour la soigner). Les descriptions et classifications des qualités de la voix et l'importance de sa couleur parcourent I'histoire de la musique autant que I'attention portée aux pigments et qualités chromatiques parcourt I'histoire de l'art - des traités médiévaux de compositions des couleurs aux recherches de Michel Pastoureaux.

$\mathrm{Si}$ « la musique est une imitation, ou représentation, aussi bien que [...] la peinture, car elle fait avec les sons, ou la voix articulée ce que le Peintre fait avec la lumière, l'ombre et les couleurs » (Traitez des chants, p. 93), le son de la voix est une représentation de l'intériorité de l'être et elle est à l'oreille ce que le visage est à la vue pour la connaissance de I'autre. "Le visage, \& la voix sont les miroirs de I'ame, qui suppleent en quelque façon à la fenestre que Momus desiroit vis à vis du coeur » (Traitez de la voix, p. 8). Si l'individu ne peut exprimer ses pensées que par I'intermédiaire de sa voix, cette interface entre l'intériorité et l'extériorité est un double miroir car le son de la voix permet également au monde extérieur de voir l'intériorité de l'être. La première voix articule des idées, la seconde peut se limiter à donner sa pure couleur car la connaissance ou la reconnaissance que le monde extérieur a de l'intériorité dépend simplement de la qualité de la voix, indépendamment des mots. La voix en elle même signifie: elle dit l'identité, les passions, leurs altérations et le tempérament. L'identité d'abord en ce que la voix qualifie I'individu dans sa singularité: Mersenne prend à plusieurs reprises l'exemple biblique d'Isaac, aveugle, trompé en touchant Jacob qui s'était travesti pour se faire passer pour son frère alors que son père reconnut sa voix (cf. Genèse 27, 22). La voix, à moins qu'elle ne soit déguisée et contrefaite dans le but de tromper I'oreille, témoigne de l'individu - c'est pourquoi Jean-Baptiste est "une » voix, et non sa voix. Reflet des passions ensuite: les cris inarticulés des animaux sont l'indice des passions qui les traversent. Les 
23 Comme Augustin, sur les traces d'Aristote, Mersenne distingue néanmoins la conscience musicale (qui n'appartient qu'aux êtres raisonnables) des sons émis par les animaux. Sur ce thème dans les Questions harmoniqueset dans le Discours sceptique sur la musique, voir Peter SzEndY : «Phtongonomie animales chez Aristote, Mersenne, Kircher», Marta Grabocs (ed.): Méthodes nouvelles, musiques nouvelles: Musicologie et création, Strasbourg, Presses Universitaires de Strasbourg, 1999, p. 11-29.

24 Cesare Ripa, dans son Iconologia (Rome, eredi di Giovanni Gigliotti, 1593), remarque la même chose: celui qui crie épouvante les oreilles alors que son visage lui aussi se contorsionne et épouvante la vue («minaccia» «nel gridare si commuove il sangue, si porta sempre un non so che di spaventevole nella faccia, \& come la voce commuove gli orecchi, così i lineamenti della faccia spaventano per la vista dispiacevole »).

25 Roland BARThes, Le plaisir du texte, Paris, Seuil, 1982 (Paris, 1973), p. 88 ; voir également Le grain de la voix. Entretiens 1962-1980, Paris, Seuil, 1981, p. 9-13; p. 200. chiens, note Mersenne, font un autre cri quand ils se fâchent ou se plaignent, quand ils sont malades ou quand ils se réjouissent (cf. Traitez de la voix, p. 8). ${ }^{23}$ La portée signifiante de la voix ne dépend donc pas des paroles: le son de la voix signifie et ses altérations disent la santé ou la maladie, ainsi que les passions internes (colère, joie). ${ }^{24}$ Passions et tempéraments enfin: "la voix est plus aiguë en la tristesse \& en la colère, que hors de ces passions, car la bile fait la voix aiguë, la melancholie, \& le phlegme la fait grave, \& I'humeur sanguin la rend temperée» (Idem). Mersenne conjugue ainsi les anciennes théories médicales grecques aux observations de la nature, les exemples bibliques à la tradition augustinienne et, par la confluence des sources, confirme combien la voix signifie au-delà et endeçà des paroles.

\section{Conclusions}

La voix, ses couleurs et modulations, ses inflexions et altérations, est objet de recherche depuis la patristique grecque jusqu'à Roland Barthes, fasciné par le grain de la voix comme pouvait l'être Grégoire de Nysse. "Le grain de la voix, qui est un mixe érotique de timbre et de langage, $[\ldots]$ peut donc être $[\ldots .$.$] la matière d'un art { }^{25}$ La matière révèle I'intériorité de l'être : la voix, avec ses altérations et sa texture, dit l'identité cachée et son rapport à l'altérit («parle, afin que ie te voye»).

L'iconologie de la voix qui se dégage des textes d'Augustin, Grégoire, Jean Scot et Mersenne se structure autour de trois niveaux comme autant d'éléments d'une architecture future - construction complétée de l'iconologie de la voix avant l'âge moderne. Le premier niveau, le plus élémentaire, est descriptif: la description des registres de la voix dans divers récits - par exemple les récits hagiographiques - est l'écho de I'image narrative. Les descriptions de la voix - du murmure au cri, du bruit au chant - participent à la théâtralisation de I'action (la mort chez Grégoire). La voix représente les passions de l'âme - celles-là même que théorise Mersenne - sans qu'il y ait aucune volonté de théorisation: le récit est dans la pure représentation théâtrale. Les récits hagiographiques offrent un type de sources au musicologue pour reconstruire l'imaginaire sonore d'une époque, ses événements, ses questionnements. Ils complètent un aspect absent des tableaux : Ia dimension acoustique qui accompagne les vies et les morts - l'iconographie des fameuses tentations diaboliques de saint Antoine montre le cortège de démons monstrueux qui assaillent le vaillant personnage, mais ne dit rien de I'univers sonore qui I'entoure et se déchaîne autour de lui. Pour avoir une 
représentation - une histoire - des cris, hurlements, déchirements de I'air, nuages éclatant de voix horribles auxquels s'opposent les harmonies suaves de paroles et de chants sacrés, il faut se tourner vers les sources littéraires (la vie de saint Antoine par Athanase d'Alexandrie), car les représentations visuelles ne rendent pas compte de l'étendue de la gamme sonore. L'historien de l'art se penche sur les toiles, tables et miniatures pour y voir les formes et les figures, et I'historien de la musique complète le tableau par l'analyse des descriptions sonores et musicales présentes dans les textes littéraires. Le second niveau quitte le récit hagiographique et il est intrinsèquement théologique. Les mots pour le dire peuvent être empruntés à la sphère philosophique, voire psychophysiologique, mais le contenu du débat est de nature théologique : la voix est la matière du Verbe. Elle est transparence - invisible - du Verbe. La voix n'est plus matière des passions, mais d'interrogations qui ont trait aux rapports entre le Père et I'incarnation du Fils. La rationalité du logos passe par la matérialité de la voix (Augustin, Jean Scot). Le troisième niveau évolue des spéculations théologiques aux spéculations philosophiques et anthropologiques: la voix est le miroir de l'âme et la matière du chant (Augustin, Mersenne). Elle dit, témoigne ou raconte imaginairement les passions de l'âme. Comme la musique, elle représente: elle met en scène par le geste sonore les passions intérieures de l'être. Interface entre l'intériorité et I'extériorité (Grégoire, Augustin, Mersenne), lieu de construction ou de dépassement de I'identité (Jean Scot), possibilité de connaissance, voire de dialogue (Augustin, Jean Scot, Mersenne), la voix donne corps à I'être et à I'autre.

Théoriciens musicaux, médecins et philosophes de la musique se sont penchés sur les couleurs de la voix et ses altérations chromatiques ou ses maladies chroniques. Pendant ce temps, les théologiens examinent les rapports de la voix au son et au verbe. Le regard porté sur les qualités de la voix renvoie aux descriptions littéraires, et les considérations médicales et éthiques sur les altérations de la voix font écho aux récits hagiographiques. Il y aurait encore une source à envisager aux confins entre médecine et théologie. Si Mersenne affirme que la voix est le miroir de I'âme et que I'on peut donc connaître quelqu'un par le son de sa voix son unicité et sa qualité sonore - c'est également une idée qui parcourt toute la littérature des traités de démonologie depuis le De operatione daemonum du philosophe byzantin Michel Psellos (1018-1078) aux traités de la fin du Xvirème siècle. Le médecin dans ses recherches de démonologie apprend à distinguer les sortes d'esprits diaboliques par le son de leurs voix. Les esprits malins se manifestent par deux types de voix: une voix obscure, grave, sombre et ténébreuse, et une autre au 
contraire légère et aiguë. À partir de ces signes sonores, le médecin peut distinguer les esprits malins, mieux connaître le mal et donc le soigner. À quelques années de distance du Traitez sur la voix et les chants de Mersenne, le traité du jésuite Petro Thyraeo (1546-1601), Daemoniaci cum locis infestis et terriculamentis nocturnis, réédité à Cologne en 1627, reprend une longue tradition de recherche sur le son et la voix, les bruits et les musiques du diable dans son chapitre "Quaeratio \& conditio Vocum, Sonorum, atque Tumuluum, qui auribus viventium percipiuntur, \& a Spectris, sive Spiritibus, locis infestis excitantur» (p. 79). Le possédé et le possédant sont tous deux définis en termes sonores. Le possédé n'est autre que clamores (p. 162): il est réduit à un langage insensé, inarticulé et irrationnel. Parallèlement, les démons le possédant sont travestissements de voix et de musiques, bruits et sonorités tant ravageuses qu'horribles. Les descriptions des voix démoniaques présentes dans les traités de démonologie constituent une source extrêmement riche pour la représentation de l'imaginaire sonore du mal et du malin : cris horribles, bruits assourdissants, hurlement strident, son obscur de la voix, langage non articulé, paroles ambiguës, fausses imitations musicales sont autant de caractéristiques de la voix et du langage démoniaques. Contemporaines aux traités musicaux renaissants, il conviendrait, ailleurs, de les étudier en tant que telles. Ces traités sont également un complément culturel aux sources musicales de la même époque et aux représentations du monde et des esprits démoniaques par exemple dans I'Opéra - depuis Orphée.

Tous - théoriciens musicaux ou hagiographes, médecins ou philosophes de la musique - tendent à voir à travers la couleur de la voix et ses altérations. À voir une passion, un affect, une maladie, un vice, une imperfection. À déterminer une identité : tempérament chez l'être humain ou espèce démoniaque. Les couleurs et altérations de la voix révèlent I'intériorité (les passions de l'âme ou les corruptions des organes internes) et en cela font voir. L'altération et donc le changement qualitatif de la voix étouffée, ténébreuse etc. - est un signe de la quantité des humeurs, de la faiblesse musculaire, de l'enflure de certains organes phonétiques, ainsi qu'un signe de l'altération de l'âme en ce qu'elle manifeste les passions - colère, peur, douleur, joie. Si chaque tempérament possède une voix qui le caractérise, les altérations de cette dernière témoignent d'un déséquilibre ou d'une corruption psychosomatique, d'un désir ou d'une passion de I'âme. De Grégoire de Nysse à Mersenne, la conscience que la voix dit indépendamment des paroles se renforce. Modulations inarticulées de la voix - gémissements, cris - ou couleur de la voix qui, sans s'appliquer au mot, manifeste le tempérament et les passions de l'âme: la voix signi- 
fie. En cela, ces textes représentent I'archéologie du savoir sémiologique et répondent à la même question que celle que pose Roland Barthes dans ses séminaires sur la voix : "Comment une voix peut-elle signifier, indépendamment de ce qu'elle dit? ».26 Tous rendent compte d'une même question paradoxale : comment se voir et se faire voir si ce n'est en parlant? Question dont la réflexivité devient double: "Comment se regarder autrement qu'en se parlant? ». ${ }^{27}$

\section{Laurence Wuidar \\ FNS - Université de Genève \\ laurence.wuidar@gmail.com}

26 Roland BARTHES: "Les fantômes de l'Opéra », Le Nouvel Observateur, 17 décembre 1973, Entretiens..., p. 200.

27 Roland BARTHES : «Que deviendrait une société qui renoncerait à se distancer? », Le Monde, 15 novembre 1974, Entretiens..., p. 213.

ICONOLOGIE de LA VOIX: DU SERMON DE VOCE ET VERBO AU TRAITEZ DE LA VOIX ET DES CHANTS

Le but de cette contribution est de tracer une iconologie de la voix dans un ensemble de textes de nature théologique et philosophique avant l'époque moderne. Le son signifie-t-il indépendamment des mots ? Le son manifeste-t-il l'intériorité de l'âme et est-il miroir de la psyché ? Le récit de Grégoire de Nysse sur la vie de sainte Macrine, le sermon De voce et verbo d'Augustin et le Commentaire sur l'évangile de saint Jean de Jean Scot Eurigène sont les trois textes analysés d'un point de vue musicologique. Grégoire offre un tableau sonore de la mort de sa sœur, introduisant le lecteur dans la sémantique du son et du cri au sein d'un récit hagiographique. Le sermon d'Augustin interroge la nature passagère du son dans son union à la permanence du Verbe, ainsi que la voix comme interface entre l'intériorité de l'être et I'extériorité du monde. Enfin, Jean Scot pénètre dans les spéculations théologiques sur la voix comme miroir de l'autre: Jean Baptiste est voix d'un autre, voix de I'altérité qui transcende son identité propre. En cela, Augustin et Jean Scot ne sont pas sans rapport avec les considérations sur la voix du Traitez de la voix et des chants (1636) de Marin Mersenne qui clôt notre analyse: la voix est miroir de l'âme, mais la voix du chanteur, comme celle de Jean, manifeste les passions d'un autre.

Mots clés: Théorie musicale, voix, identité, altérité, passions, signification, son, bruit, langage, Grégoire de Nysse, Augustin, Jean Scot Eurigène, Mersenne.

ICONOLOGy OF VOICE: FROM DE VOCE ET VERBO TO TRAITEZ DE LA VOIX ET DES CHANTS

Iconology of voice is investigated through philosophical and theological texts. The treatises of Gregory of Nyssa's Life of St. Macrina, Augustine's De voce et verbo and Jean Scot's Commentary on St. John are analyzed from a musicological point of view. Does voice - and thus sound without words - have any significance? Is sound without words the language and manifestation of psyche? Gregory offers a sonorous picture of the death of his sister in which music, silence, cries and screams are described. Augustine questions the difference between the fragility of sound and the eternity of the word a well as the incarnation of Logos in temporary sounds and voice as the interface between inner life and the external world. Jean Scot, philosophical heir to both Gregory and Augustine, proposes a profound speculation on voice, as the manifestation of otherness. John the Baptist is the voice of another, he, whose identity is to be the voice, is the incarnation of somebody else. This theological speculation finds an echo in Marin Mersenne's philosophical and musical 
ideas. In his Traitez de la voix et des chants (1636) the philosopher exemplifies the voice as the mirror of the soul, thus reflecting all inner passions. Concurrently he also underlines that the musical voice embodies the passions of someone else. Therefore as John, the singer, is the voice of some one else it is not his voice and does not manifest his own passions. In this article unusual sources are analyzed, as we find that from late antiquity to the first half of the seventeenth century diverse theological discourses contain musical speculations.

Keywords: music theory, voice, identity, otherness, ethos theory, sign and signification, sound, rumor, language, Gregory of Nyssa, Augustine, Jean Scot, Mersenne. 